\title{
Differential Susceptibility of Tree Tomato (Solanum betaceum) Cultivars to Late Blight Caused by Phytophthora betacei
}

\author{
Maria F. Mideros, ${ }^{1}$ Hilary Mayton, ${ }^{2}$ Giovanna Danies, ${ }^{3}$ Luz E. Lagos, ${ }^{4}$ William E. Fry, ${ }^{2}$ and Silvia Restrepo ${ }^{1, \dagger}$ \\ ${ }^{1}$ Department of Biological Sciences, Universidad de los Andes, Bogotá, Colombia \\ ${ }^{2}$ School of Integrative Plant Science, Cornell University, Ithaca, NY, U.S.A. \\ ${ }^{3}$ Department of Design, Universidad de los Andes, Bogotá, Colombia \\ ${ }^{4}$ Department of Biology, Universidad de Nariño, Pasto, Colombia
}

\begin{abstract}
Host-pathogen interactions of a new species of Phytophthora, causal agent of late blight of tree tomato (Solanum betaceum Cav.), identified as Phytophthora betacei, were investigated with four different cultivars. Thirty-six P. betacei isolates, collected from southern Colombia between 2008 and 2009, were used to inoculate common tree tomato cultivars, Común, Híbrido, Injerto, and Holandés. Data on incubation and latent periods as well as infection efficiency, lesion development, and total sporulation were collected via detached leaf assays. Significant differences in susceptibility, based on the parameters measured, were observed. Común was the most susceptible cultivar, followed by Injerto, Híbrido, and Holandés. The mean incubation period was lowest for Común at

produced necrotic lesions on Común, and 33, 24, and 21 caused infection on Injerto, Híbrido, and Holandés, respectively. Two isolates were able to cause infection only on Común, and 13 isolates were able to infect al four cultivars. Infection efficiency was significantly higher for the cultivar Común, followed by Injerto, Híbrido, and Holandés. Average lesion size was larger on Común than on any other cultivar. An inverse relationship of lesion size and total sporulation was observed. Común had significantly lower total sporulation than Híbrido and Holandés, which had the smallest average lesion sizes. These data show variation in pathogenicity of $P$. betacei isolates, under controlled conditions, and differential susceptibility of four distinct $S$. betaceum cultivars.
\end{abstract} $125.6 \mathrm{~h}$ post-inoculation (hpi) and highest for Híbrido at $139.4 \mathrm{hpi}$. No significant differences in latent period were observed. All 36 isolates
Keywords: late blight, Phytophthora betacei, tree tomato
The plant pathogen Phytophthora betacei Mideros, L.E. Lagos \& $\mathrm{S}$. Restrepo, has recently been described as a new species and the causal agent of late blight disease on tree tomato (Solanum betaceum Cav.) in Colombia (Mideros et al. 2018). Tree tomato or tamarillo is a small, fast-growing tree that produces egg-shaped edible fruits and is native to the Andean region of South America (Acosta-Quezada et al. 2011; Bonnet and Cárdenas 2012; Murillo-Gómez et al. 2017). Tamarillo has been identified as a promising crop for production of small nutritious fruits in many tropical and subtropical regions of the world. The crop is cultivated worldwide, and fruits are exported across the globe, including European and North American markets, with some production in New Zealand (Murillo-Gómez et al. 2017; Prohens and Nuez 2001;). The fruit, high in antioxidants and nutritional value, is consumed raw or can be used in jams or preserves and juices (Espin et al. 2016; Nor et al. 2018). Tree tomatoes are self-compatible and can be propagated by seeds or cuttings, with fruit production beginning in the first or second year after planting (Murillo-Gómez et al. 2017). Individual trees average 3.5 to $5 \mathrm{~m}$ in height and have an average life span of 5 to 12 years. Classification of tree tomato accessions or cultivars has historically been based on fruit shape and color (Acosta-Quezada et al. 2011). There has been very little plant breeding for tree tomatoes, and some cultivar groups may have only one or two genotypes available for commercial production. Breeding material is limited, but increases in yield and pathogen resistance

${ }^{\dagger}$ Corresponding author: S. Restrepo; srestrep@uniandes.edu.co

Funding: This research was supported by the Department of Biological Sciences at Universidad de los Andes. Additional funding for this research was provided by the Research Fund of the School of Sciences and the Office of the Vice Presidency for Research from Universidad de los Andes.

The author(s) declare no conflict of interest.

Accepted for publication 3 November 2019.

(C) 2020 The American Phytopathological Society may be obtained via hybrid crosses with Solanum unilobum (EncisoRodriguez et al. 2010).

In Colombia, tree tomato production occurs primarily in the states of Nariño, Caldas, Cundinamarca, and Antioquia. Anthracnose caused by Colletotrichum species has recently been reported to cause significant losses in yield (Pardo-De la Hoz et al. 2016; Rojas et al. 2018), and late blight epidemics on tree tomatoes have increased in the southern region of Colombia and in Peru and Ecuador (Forbes et al. 2016; Mideros et al. 2018). Characterization of Phytophthora sp. isolated from late blight-like lesions from wild tree tomatoes, and commercial plantations in diverse regions of Colombia, Peru, and Ecuador revealed genetically distinct populations (Forbes et al. 2016). Peruvian isolates of Phytophthora andina differed from populations isolated from tree tomato lesions in Colombia and Ecuador based on restriction fragment length polymorphism (RFLP), mitochondrial haplotype, mating type, and simple sequence repeat (SSR) genetic markers (Forbes et al. 2016). In the Andes region, $P$. andina, Phytophthora infestans sensu lato, and $P$. betacei have all been identified as causal agents of late blight or late blight-like disease on tree tomato (Adler et al. 2004; Mideros et al. 2018; Oliva et al. 2010). The characterization and systematic classification of Phytophthora species isolated from commercial crops of tomato (Solanum lycopersicum L.) and potato (Solanum tuberosum L.) and wild Solanum species from the Andean highlands have resulted in the identification of new species and clonal lineages with many undefined pathogenicity traits (Adler et al. 2004; Martin et al. 2012; Mideros et al. 2018; Oliva et al. 2010; Ordonez et al. 2000).

Previous research with $P$. betacei has shown that tree tomato is the only known host of this newly described plant pathogen because it is unable to colonize potatoes or tomatoes in the field (Mideros et al. 2018). The susceptibility of different tree tomato cultivar groups to late blight and variation in the $P$. betacei pathogen population is largely unknown. Key pathogenicity traits, such as infection efficiency, incubation and latent periods, sporulation, and lesion development on diverse tree tomato genotypes have not been elucidated in this pathosystem. As production increases in Colombia and other regions of the world, it is important to obtain disease resistance and host-pathogen data to develop integrated management strategies for commercial producers. In this study, we investigated the 
differential pathogenicity of $P$. betacei on four cultivars of its natural host, S. betaceum, by using detached leaf assays. For this purpose, we inoculated $36 P$. betace $i$ isolates under controlled conditions.

\section{Materials and Methods}

Pathogen strains. A total of $36 P$. betacei isolates were used in these assays (Table 1). All isolates were collected in southern Colombia between 2008 and 2009 by Mideros et al. (2018) (Table 1). Isolates were recovered from the Phytophthora collection of Universidad de los Andes for these experiments and were maintained at $18^{\circ} \mathrm{C}$ on a selective medium prepared with tree tomato fruit $\left(0.25 \mathrm{~g}_{\text {of }} \mathrm{CaCO}_{3}, 0.5 \mathrm{~g}\right.$ of yeast extract, $25 \mathrm{~g}$ of sucrose, $15 \mathrm{~g}$ of agar, and $100 \mathrm{ml}$ of tree tomato extract, composed of $550 \mathrm{~g}$ of tree tomato fruit per liter of water) (Mideros et al. 2018). The P. betacei isolates evaluated in this study belong to the 1c clade of the Phytophthora genus, very closely related to $P$. infestans and $P$. andina. In addition, RFLP analysis indicated that they belong to the EC 3 clonal lineage (Mideros et al. 2018). Variation in mitochondrial haplotype and mating type has been reported in clade 1c (Mideros et al. 2018); however, all these isolates had the same mitochondrial haplotype and mating type (Table 1).Genetic diversity of isolates determined by SSRs previously described in Mideros et al. (2018) revealed little variation within the $P$. betacei isolates (Table 2). Although there was some variation at one or two SSR alleles, the population used for this study appears to be of a single clonal lineage.

Cultivar selection. Four common cultivars of tree tomato grown in Colombia, known as Común, Híbrido, Injerto, and Holandés, were selected for evaluation. Traditional farmers in Colombia extensively cultivate these four cultivars. The most widely cultivated is the Común cultivar, which has yellow-orange pulp and reddish-orange exocarp. The Injerto cultivar has a brilliant and red exocarp with red-purple pulp. The Híbrido has orange-yellow exocarp with orange purple pulp. Finally, Holandés has the largest fruit, with reddishyellow exocarp and yellow-orange pulp.

The plant material from tree tomato cultivars used in this study was maintained in field plots located at the town of Sibundoy (Putumayo Department, coordinates $1^{\circ} 12^{\prime} 12^{\prime \prime} \mathrm{N} 76^{\circ} 55^{\prime} 9^{\prime \prime} \mathrm{W}$ ). All cultivars were grown under natural light conditions, with an average annual rainfall of approximately $1,741 \mathrm{~mm}$ per year and with a mean maximum temperature of $20.5^{\circ} \mathrm{C}$ and a minimum of $15^{\circ} \mathrm{C}$.

Experimental design and inoculation conditions. The second and third leaves (of similar size) from mature tree tomato plants (6 to 8 weeks old), of each cultivar, respectively, were collected and subsequently transferred to the laboratory in watertight boxes to prevent drying before inoculation. Each $P$. betacei strain described in Table 1 was inoculated on two different leaves from each tree tomato cultivar. Three independent blocks were evaluated, for a total of six inoculated leaves per isolate and tree tomato cultivar. Each leaf was placed abaxial side up on the base of a petri plate $(90 \mathrm{~mm})$ containing a moist paper towel. Each leaf was inoculated on four points, two on each side of the main vein. Each inoculation point consisted of a $20 \mu \mathrm{l}$ drop of a sporangial suspension $\left(3.5 \times 10^{4}\right.$ sporangia $\left./ \mathrm{ml}^{-1}\right)$, which had been previously exposed to $4^{\circ} \mathrm{C}$ for a period of $4 \mathrm{~h}$ to

Table 1. Isolate ID, collection date, global positioning system (GPS) locations, mitochondrial haplotype (mtDNA), and mating type (MT) of Phytophthora betacei isolates evaluated in this study. All isolates were obtained from tamarillo (Solanum betaceum) in Colombia

\begin{tabular}{|c|c|c|c|c|c|c|}
\hline ID & Collection date & State & Municipality & GPS location & mtDNA $^{y}$ & MT $^{\mathbf{z}}$ \\
\hline 8001 & Sep-08 & Putumayo & Santiago & $01^{\circ} 07.381^{\prime} \mathrm{N} 77^{\circ} 00.616^{\prime} \mathrm{W}$ & Ia & IA \\
\hline 8010 & Sep-08 & Putumayo & Colón & $01^{\circ} 11.094^{\prime} \mathrm{N} 76^{\circ} 58.354^{\prime} \mathrm{W}$ & Ia & IA \\
\hline 8050 & Oct-08 & Putumayo & Colón & $01^{\circ} 11.750^{\prime} \mathrm{N} 76^{\circ} 58.337^{\prime} \mathrm{W}$ & Ia & IA \\
\hline 8054 & Oct-08 & Putumayo & Santiago & $01^{\circ} 10.651^{\prime} \mathrm{N} 76^{\circ} 59.389^{\prime} \mathrm{W}$ & Ia & IA \\
\hline 8056 & Oct-08 & Putumayo & Santiago & $01^{\circ} 10.542^{\prime} \mathrm{N} 76^{\circ} 59.406^{\prime} \mathrm{W}$ & Ia & IA \\
\hline 8064 & Oct-08 & Putumayo & Santiago & $01^{\circ} 10.257^{\prime} \mathrm{N} 76^{\circ} 59.413^{\prime} \mathrm{W}$ & Ia & IA \\
\hline 8071 & Oct-08 & Putumayo & Colón & $01^{\circ} 11.967^{\prime} \mathrm{N} 76^{\circ} 57.543^{\prime} \mathrm{W}$ & Ia & IA \\
\hline 8073 & Oct-08 & Putumayo & Colón & $01^{\circ} 12.009^{\prime} \mathrm{N} 76^{\circ} 57.578^{\prime} \mathrm{W}$ & Ia & IA \\
\hline 8075 & Oct-08 & Putumayo & Colón & $01^{\circ} 13.100^{\prime} \mathrm{N} 76^{\circ} 56.455^{\prime} \mathrm{W}$ & Ia & IA \\
\hline 8076 & Oct-08 & Putumayo & Colón & $01^{\circ} 13.050^{\prime} \mathrm{N} 76^{\circ} 56.475^{\prime} \mathrm{W}$ & Ia & IA \\
\hline 8077 & Oct-08 & Putumayo & Colón & $01^{\circ} 12.949^{\prime} \mathrm{N} 76^{\circ} 56.494^{\prime} \mathrm{W}$ & Ia & IA \\
\hline 8080 & Oct-08 & Putumayo & Colón & $01^{\circ} 12.885^{\prime} \mathrm{N} 76^{\circ} 56.572^{\prime} \mathrm{W}$ & Ia & IA \\
\hline 8083 & Oct-08 & Putumayo & Colón & $01^{\circ} 13.269^{\prime} \mathrm{N} 76^{\circ} 56.739^{\prime} \mathrm{W}$ & Ia & IA \\
\hline 8087 & Nov-08 & Putumayo & San Francisco & $01^{\circ} 10.629^{\prime} \mathrm{N} 76^{\circ} 52.694^{\prime} \mathrm{W}$ & Ia & IA \\
\hline 8093 & Nov-08 & Putumayo & San Francisco & $01^{\circ} 09.426^{\prime} \mathrm{N} 76^{\circ} 54.223^{\prime} \mathrm{W}$ & Ia & IA \\
\hline 8094 & Nov- 08 & Putumayo & San Francisco & $01^{\circ} 09.426^{\prime} \mathrm{N} 76^{\circ} 54.223^{\prime} \mathrm{W}$ & Ia & IA \\
\hline 8098 & Nov-08 & Putumayo & San Francisco & $01^{\circ} 09.450^{\prime} \mathrm{N} 76^{\circ} 54.100^{\prime} \mathrm{W}$ & Ia & IA \\
\hline 9001 & Jan-09 & Nariño & Buesaco & $01^{\circ} 18.801^{\prime} \mathrm{N} 77^{\circ} 08.470^{\prime} \mathrm{W}$ & Ia & IA \\
\hline 9003 & Jan-09 & Nariño & Buesaco & $01^{\circ} 18.801^{\prime} \mathrm{N} 77^{\circ} 08.470^{\prime} \mathrm{W}$ & Ia & IA \\
\hline 9007 & Jan-09 & Nariño & Buesaco & $01^{\circ} 18.639^{\prime} \mathrm{N} 77^{\circ} 08.662^{\prime} \mathrm{W}$ & Ia & IA \\
\hline 9008 & Jan-09 & Nariño & Buesaco & $01^{\circ} 18.665^{\prime} \mathrm{N} 77^{\circ} 08.615^{\prime} \mathrm{W}$ & Ia & IA \\
\hline 9011 & Jan-09 & Nariño & Buesaco & $01^{\circ} 18.926^{\prime} \mathrm{N} 77^{\circ} 08.573^{\prime} \mathrm{W}$ & Ia & IA \\
\hline 9013 & Jan-09 & Nariño & Buesaco & $01^{\circ} 19.074^{\prime} \mathrm{N} 77^{\circ} 08.553^{\prime} \mathrm{W}$ & Ia & IA \\
\hline 9015 & Jan-09 & Nariño & Buesaco & $01^{\circ} 19.239^{\prime} \mathrm{N} 77^{\circ} 08.574^{\prime} \mathrm{W}$ & Ia & IA \\
\hline 9023 & Jan-09 & Nariño & Buesaco & $01^{\circ} 20.239^{\prime} \mathrm{N} 77^{\circ} 10.498^{\prime} \mathrm{W}$ & Ia & IA \\
\hline 9025 & Jan-09 & Nariño & Buesaco & $01^{\circ} 20.277^{\prime} \mathrm{N} 77^{\circ} 10.490^{\prime} \mathrm{W}$ & Ia & IA \\
\hline 9029 & Jan-09 & Nariño & Buesaco & $01^{\circ} 20.026^{\prime} \mathrm{N} 77^{\circ} 10.538^{\prime} \mathrm{W}$ & Ia & IA \\
\hline 9035 & Feb-09 & Nariño & Buesaco & $01^{\circ} 17.385^{\prime} \mathrm{N} 77^{\circ} 10.432^{\prime} \mathrm{W}$ & Ia & IA \\
\hline 9057 & Mar-09 & Nariño & Consaca & $01^{\circ} 12.528^{\prime} \mathrm{N} 77^{\circ} 27.336^{\prime} \mathrm{W}$ & Ia & IA \\
\hline 9070 & Mar-09 & Nariño & Buesaco & $01^{\circ} 20.239^{\prime} \mathrm{N} 77^{\circ} 27.336^{\prime} \mathrm{W}$ & Ia & IA \\
\hline 9114 & Jan-09 & Putumayo & San Francisco & $01^{\circ} 09.680^{\prime} \mathrm{N} 76^{\circ} 54.273^{\prime} \mathrm{W}$ & Ia & IA \\
\hline 9120 & Jan-09 & Putumayo & San Francisco & $01^{\circ} 10.420^{\prime} \mathrm{N} 76^{\circ} 56.225^{\prime} \mathrm{W}$ & Ia & IA \\
\hline 9128 & Jan-09 & Putumayo & Sibundoy & $01^{\circ} 11.225^{\prime} \mathrm{N} 76^{\circ} 56.740^{\prime} \mathrm{W}$ & Ia & IA \\
\hline 9147 & Feb-09 & Putumayo & Sibundoy & $01^{\circ} 10.491^{\prime} \mathrm{N} 76^{\circ} 55.413^{\prime} \mathrm{W}$ & Ia & IA \\
\hline 9151 & Feb-09 & Putumayo & Sibundoy & $01^{\circ} 11.228^{\prime} \mathrm{N} 76^{\circ} 55.413^{\prime} \mathrm{W}$ & $\mathrm{Ia}$ & IA \\
\hline 9153 & Feb-09 & Putumayo & Sibundoy & $01^{\circ} 12.315^{\prime} \mathrm{N} 76^{\circ} 55.249^{\prime} \mathrm{W}$ & Ia & IA \\
\hline
\end{tabular}

${ }^{\mathrm{y}}$ Mitochondrial haplotype was determined via PCR restriction fragment length polymorphism method, with reference strains US-1 and US-8 included as controls.

${ }^{\mathrm{z}}$ Mating type was determined via in vitro mating crosses of $P$. betacei isolates and $P$. infestans strain of the A2 mating type (US040009) (Mideros et al. 2018). 
induce zoospore release. Leaves were incubated at $15^{\circ} \mathrm{C}$ in a fully randomized block design with a 16-h light period. All experimental blocks were maintained in separate plastic boxes under the same conditions for each assay. Two additional leaves of each host cultivar were inoculated with distilled water and used as negative controls.

Assessment of pathogen traits related to host susceptibility. Infection efficiency was estimated as the percentage of successful infections by counting the number of successful infection points per leaf and dividing this number by the total number of inoculated points (four total). The lesion size (in square centimeters) was calculated as the infected area of leaf tissue at the end of the experiment (9 days post-inoculation [dpi]). Lesion size was measured from images obtained with a digital camera and importing the images into the ImageJ software (https://imagej.nih.gov/ij/). Incubation period, measured in hours post-inoculation (hpi), was calculated as the interval between inoculation and the first appearance of necrotic or lesionlike disease symptoms. The incubation period was assessed visually through a stereoscope. Latent period was measured in hours as the time interval between inoculation and the first appearance of sporangia. Sporangia production was determined as the number of sporangia produced per leaf, 9 dpi. Sporangia were dislodged from a leaflet by submerging it into $5 \mathrm{ml}$ of distilled water, and the number of sporangia was determined with a hemocytometer. Sporangial counts were repeated at least two times.

Statistical analyses. All nontransformed raw data from the host-pathogen interaction assays (lesion size, infection efficiency, sporulation, incubation, and latent periods) were evaluated for normality, homoscedasticity, and goodness of fit in Proc UnivariateNormal statistical software version 9.3, along with generation of histograms of the distribution of residuals and normal probability plots. Because of their nonnormal distributions, lesion size data were subjected to square root transformation for analysis, and infection efficiency data were transformed via arcsine square root for analysis. Sporulation, incubation, and latent periods showed normal distributions and were not transformed for further analyses. Analysis of variance (ANOVA, in PROC GLM, statistical software version 9.3) was used to evaluate statistical significance of differences of means for host-pathogen interactions via Fisher LSD test at a significance level of $\alpha=0.05$. ANOVAs were conducted on both transformed and nontransformed data for lesion size and infection efficiency. In addition, Proc Freq $/ \chi^{2}$ was used to evaluate cultivar type and the number of isolates able to cause infection in the detached leaf assays. There were no differences in ANOVAs for significance of mean separation between nontransformed and transformed data for lesion size and

Table 2. Simple sequence repeat $(\mathrm{SSR})^{\mathrm{z}}$ multilocus genotypes of Phytophthora betacei isolates used in this study and isolates of Phytophthora andina lineage and Phytophthora infestans to allow comparisons across species

\begin{tabular}{|c|c|c|c|c|c|c|c|c|c|c|c|c|}
\hline Isolate & Species & D13 & G11 & Pin04 & Pin33 & Pin56 & Pin70 & Pin89 & $4 B$ & Pin63 & Pin02 & Pin16 \\
\hline 8001 & P. betacei & 104 & $130 / 160$ & $166 / 170$ & 203 & $174 / 176$ & 195 & 185 & $213 / 273 / 278$ & $148 / 151$ & 160 & $176 / 178$ \\
\hline 8010 & P. betacei & 104 & $130 / 160$ & $166 / 170$ & 203 & $174 / 176$ & 195 & 185 & $213 / 278 / 285$ & $148 / 151$ & 160 & $176 / 178$ \\
\hline 8050 & P. betacei & 104 & $130 / 160$ & $166 / 170$ & 203 & $174 / 176$ & 195 & 185 & $213 / 273 / 278$ & $148 / 151$ & 160 & $176 / 178$ \\
\hline 8054 & P. betacei & 104 & $130 / 160$ & $166 / 170$ & 203 & $174 / 176$ & 195 & 185 & $213 / 269 / 278$ & $148 / 151$ & 160 & $176 / 178$ \\
\hline 8056 & P. betacei & 104 & $130 / 156$ & $166 / 170$ & 203 & $174 / 176$ & 195 & 185 & $213 / 273 / 278$ & $148 / 151$ & 160 & $176 / 178$ \\
\hline 8064 & P. betacei & 104 & $130 / 160$ & $166 / 170$ & 203 & $174 / 176$ & 195 & 185 & $213 / 269 / 278$ & $148 / 151$ & 160 & $176 / 178$ \\
\hline 8071 & P. betacei & 104 & $130 / 160$ & $166 / 170$ & 203 & $174 / 176$ & 195 & 185 & $213 / 278 / 285$ & $148 / 151$ & 160 & $176 / 178$ \\
\hline 8073 & P. betacei & 104 & $130 / 156$ & $166 / 170$ & 203 & $174 / 176$ & 195 & 185 & $213 / 273 / 278$ & $148 / 151$ & 160 & $176 / 178$ \\
\hline 8075 & P. betacei & 104 & $130 / 160$ & $166 / 170$ & 203 & $174 / 176$ & 195 & 185 & $213 / 269 / 278 / 285$ & $148 / 151$ & 160 & $176 / 178$ \\
\hline 8076 & P. betacei & 104 & $130 / 156$ & $166 / 170$ & 203 & $174 / 176$ & 195 & 185 & $213 / 273 / 278$ & $148 / 151$ & 160 & $176 / 178$ \\
\hline 8077 & P. betacei & 104 & $130 / 160$ & $166 / 170$ & 203 & $174 / 176$ & 195 & 185 & $213 / 273 / 278$ & $148 / 151$ & 160 & $176 / 178$ \\
\hline 8080 & P. betacei & 104 & $130 / 156$ & 170 & 203 & $174 / 176$ & 195 & 185 & $213 / 273 / 278$ & $148 / 151$ & 160 & $176 / 178$ \\
\hline 8083 & P. betacei & 104 & $130 / 158$ & $166 / 170$ & 203 & $174 / 176$ & 195 & 185 & $213 / 273 / 278$ & $148 / 151$ & 160 & $176 / 178$ \\
\hline 8087 & P. betacei & 104 & $130 / 160$ & $166 / 170$ & 203 & $174 / 176$ & 195 & 185 & $213 / 273 / 278$ & $148 / 151$ & 160 & $176 / 178$ \\
\hline 8093 & P. betacei & 104 & 130 & $166 / 170$ & 203 & $174 / 176$ & 195 & 185 & $213 / 273 / 278$ & $148 / 151$ & 160 & $176 / 178$ \\
\hline 8094 & P. betacei & 104 & $130 / 156$ & $166 / 170$ & 203 & $174 / 176$ & 195 & 185 & $213 / 273 / 278$ & $148 / 151$ & 160 & $176 / 178$ \\
\hline 8098 & P. betacei & 104 & $130 / 156$ & $166 / 170$ & 203 & $174 / 176$ & & 185 & $213 / 273 / 278$ & $148 / 151$ & 160 & $176 / 178$ \\
\hline 9001 & P. betacei & 104 & $130 / 156$ & $166 / 170$ & 203 & $174 / 176$ & 195 & 185 & $213 / 273 / 278$ & $148 / 151$ & 160 & $176 / 178$ \\
\hline 9003 & P. betacei & 104 & $130 / 160$ & $166 / 170$ & 203 & $174 / 176$ & 195 & 185 & $213 / 273 / 285$ & $148 / 151$ & 160 & $176 / 178$ \\
\hline 9007 & P. betacei & 104 & $130 / 156$ & $166 / 170$ & 203 & $174 / 176$ & 195 & 185 & $213 / 273 / 278$ & $148 / 151$ & 160 & $176 / 178$ \\
\hline 9008 & P. betacei & 104 & $130 / 160$ & $166 / 170$ & 203 & $174 / 176$ & 195 & 185 & $213 / 278 / 285$ & $148 / 151$ & 160 & $176 / 178$ \\
\hline 9011 & P. betacei & 104 & $130 / 156$ & $166 / 170$ & 203 & $174 / 176$ & 195 & 185 & $213 / 269 / 273 / 278$ & $148 / 151$ & 160 & $176 / 178$ \\
\hline 9013 & P. betacei & 104 & $130 / 156$ & $166 / 170$ & 203 & $174 / 176$ & 195 & 185 & $213 / 273 / 278$ & $148 / 151$ & 160 & $176 / 178$ \\
\hline 9015 & P. betacei & 104 & $130 / 160$ & $166 / 170$ & 203 & $174 / 176$ & 195 & 185 & $213 / 273 / 278$ & $148 / 151$ & 160 & $176 / 178$ \\
\hline 9023 & P. betacei & 104 & $130 / 156$ & $166 / 170$ & 203 & $174 / 176$ & 195 & 185 & $213 / 269 / 278 / 285$ & $148 / 151$ & 160 & $176 / 178$ \\
\hline 9025 & P. betacei & 104 & $130 / 160$ & $166 / 170$ & 203 & $174 / 176$ & 195 & 185 & $213 / 269 / 273 / 285$ & $148 / 151$ & 160 & $176 / 178 / 182$ \\
\hline 9029 & P. betacei & 104 & $130 / 160$ & $166 / 170$ & 203 & $174 / 176$ & 195 & 185 & $213 / 269 / 273 / 278$ & $148 / 151$ & 160 & $176 / 178$ \\
\hline 9035 & P. betacei & 104 & $130 / 160$ & $166 / 170$ & 203 & $174 / 176$ & 195 & 185 & $213 / 273 / 285$ & $148 / 151$ & 160 & $176 / 178$ \\
\hline 9057 & P. betacei & 104 & $130 / 160$ & $166 / 170$ & 203 & $174 / 176$ & 195 & 185 & $213 / 273 / 285$ & $148 / 151$ & 160 & $176 / 178$ \\
\hline 9070 & P. betacei & 104 & $130 / 160$ & $166 / 170$ & 203 & 174 & 195 & 185 & 213 & $148 / 151$ & 158 & $176 / 178$ \\
\hline 9114 & P. betacei & 104 & $130 / 160$ & $166 / 170$ & 203 & $174 / 176$ & 195 & 185 & $213 / 269 / 273 / 278$ & $148 / 151$ & 160 & $176 / 178$ \\
\hline 9120 & P. betacei & 104 & $130 / 156$ & $166 / 170$ & 203 & $174 / 176$ & 195 & 185 & $213 / 273 / 278$ & $148 / 151$ & 160 & $176 / 178$ \\
\hline 9128 & P. betacei & 104 & $130 / 158$ & $166 / 170$ & 203 & $174 / 176$ & 195 & 185 & $213 / 273 / 278$ & $148 / 151$ & 160 & $176 / 178$ \\
\hline 9147 & P. betacei & 104 & $130 / 160$ & $166 / 170$ & 203 & $174 / 176$ & 195 & 185 & $213 / 273 / 278$ & $148 / 151$ & 160 & $176 / 178$ \\
\hline 9151 & P. betacei & 104 & $130 / 158$ & $166 / 170$ & 203 & $174 / 176$ & 195 & 185 & $213 / 273 / 278$ & $148 / 151$ & 160 & $176 / 178$ \\
\hline 9153 & P. betacei & 104 & $130 / 160$ & $166 / 170$ & 203 & $174 / 176$ & 195 & 185 & $213 / 273 / 278$ & $148 / 151$ & 160 & $176 / 178$ \\
\hline EC3163 & P. andina & $104 / 150 / 152$ & $130 / 202$ & 170 & 203 & $174 / 176$ & & 183 & $209 / 247 / 263$ & $148 / 151$ & 158 & $176 / 178$ \\
\hline EC3189 & P. andina & 104 & $128 / 202$ & $166 / 170$ & 203 & $174 / 176$ & 195 & 183 & $209 / 247 / 287$ & $148 / 151$ & 160 & $176 / 186$ \\
\hline EC3678 & P. andina & $104 / 150$ & $130 / 202$ & 170 & 203 & $174 / 176$ & & 183 & $209 / 251 / 263$ & $148 / 151$ & 160 & $176 / 178$ \\
\hline EC3780 & P. andina & 138 & $128 / 200$ & $166 / 170$ & 203 & 174 & 195 & 183 & $213 / 247 / 263$ & $148 / 151$ & 160 & $176 / 178$ \\
\hline EC3818 & $P$. andina & 104 & $128 / 160$ & 170 & 203 & 176 & 195 & 185 & $209 / 247 / 263 / 285$ & $148 / 151$ & 160 & $176 / 178$ \\
\hline US040009 & P. infestans & 104 & 156 & $166 / 170$ & 203 & 176 & 195 & 183 & $213 / 225$ & 157 & $160 / 162$ & $174 / 178$ \\
\hline US940494 & P. infestans & 144 & $132 / 156$ & $166 / 170$ & 203 & 176 & 195 & 179 & 213 & 157 & $152 / 160$ & 178 \\
\hline
\end{tabular}

${ }^{\mathrm{z}}$ SSR profiles used in this study were previously described in Mideros et al. (2018). 
infection efficiency. Therefore, nontransformed means are presented in Table 3 for all host-pathogen interactions evaluated.

\section{Results}

Assessment of pathogen traits related to host susceptibility. Significant differences in overall host susceptibility of tree tomato cultivars, as measured by the different parameters on detached leaves, were observed (Fig. 1). No lesion development was observed on the leaves inoculated with distilled water. All P. betacei isolates tested were able to cause disease on the cultivar Común, whereas the cultivars Injerto, Híbrido, and Holandés were infected by only some $P$. betace $i$ isolates (Fig. 2). Thirty-three, 24, and 21 isolates of $P$. betace $i$ were able to cause infection on cultivars Injerto, Híbrido, and Holandés, respectively. The most tolerant cultivar was Holandés: 15 isolates were not able to infect this genotype. Variation in infectivity of isolates was observed as 13 isolates were able to infect all four cultivars, and only two isolates (8050 and 9013) were able to infect only one tree tomato cultivar (Fig. 3). Comparison by $\chi^{2}$ showed a significant effect for cultivar type and the number of isolates able to cause infection in the detached leaf assays $\left(\chi^{2}=25.77, P<0.0001\right)$. Incubation period was significantly lower for Común than Híbrido but was not significantly different from the other three cultivars evaluated (Table 3). No significant differences were found for latent period. Infection efficiency was significantly different in all four cultivars evaluated. The highest infection efficiency, recorded by lesion development and sporulation in response to inoculations with $P$. betacei, was observed in Común (59.9\%) followed by Injerto, Híbrido, and Holandés with 27.7, 17.2, and 10.2

Table 3. Results of the evaluation of 36 isolates of Phytophthora betacei inoculated on four cultivars of tree tomato on lesion size, infection efficiency, sporulation, incubation and latent periods in detached leaf laboratory assays ${ }^{\mathrm{z}}$

\begin{tabular}{lccccc}
\hline Cultivar & $\begin{array}{c}\text { Lesion } \\
\text { size }\left(\mathbf{c m}^{\mathbf{2}}\right)\end{array}$ & $\begin{array}{c}\text { Infection } \\
\text { efficiency } \\
(\boldsymbol{\%})\end{array}$ & $\begin{array}{c}\text { Spore } \\
\text { production }\end{array}$ & $\begin{array}{c}\text { Incubation } \\
\text { period }\end{array}$ & $\begin{array}{c}\text { Latent } \\
\text { period }\end{array}$ \\
\hline Común & $9.7 \mathrm{a}$ & $59.9 \mathrm{a}$ & $17,592 \mathrm{~b}$ & $125.6 \mathrm{~b}$ & $147.2 \mathrm{a}$ \\
Injerto & $8.9 \mathrm{ab}$ & $27.7 \mathrm{~b}$ & $23,311 \mathrm{ab}$ & $136.1 \mathrm{ab}$ & $155.7 \mathrm{a}$ \\
Híbrido & $7.5 \mathrm{bc}$ & $17.2 \mathrm{c}$ & $27,747 \mathrm{a}$ & $139.4 \mathrm{a}$ & $156.7 \mathrm{a}$ \\
Holandés & $7.1 \mathrm{c}$ & $10.2 \mathrm{~d}$ & $25,830 \mathrm{a}$ & $135.8 \mathrm{ab}$ & $157.0 \mathrm{a}$ \\
$P$ & 0.004 & 0.001 & 0.003 & 0.011 & $\mathrm{NS}$ \\
\hline
\end{tabular}

${ }^{\mathrm{z}}$ Infection efficiency was estimated as the percentage of successful infections by counting the number of successful infection points per leaf and dividing this number by the total number of inoculated points (four total). Analysis of variance was used to evaluate statistical differences using Fisher LSD test at a significance level of $\alpha=0.05$. Means with the same letters are not significantly different. NS = not significant.

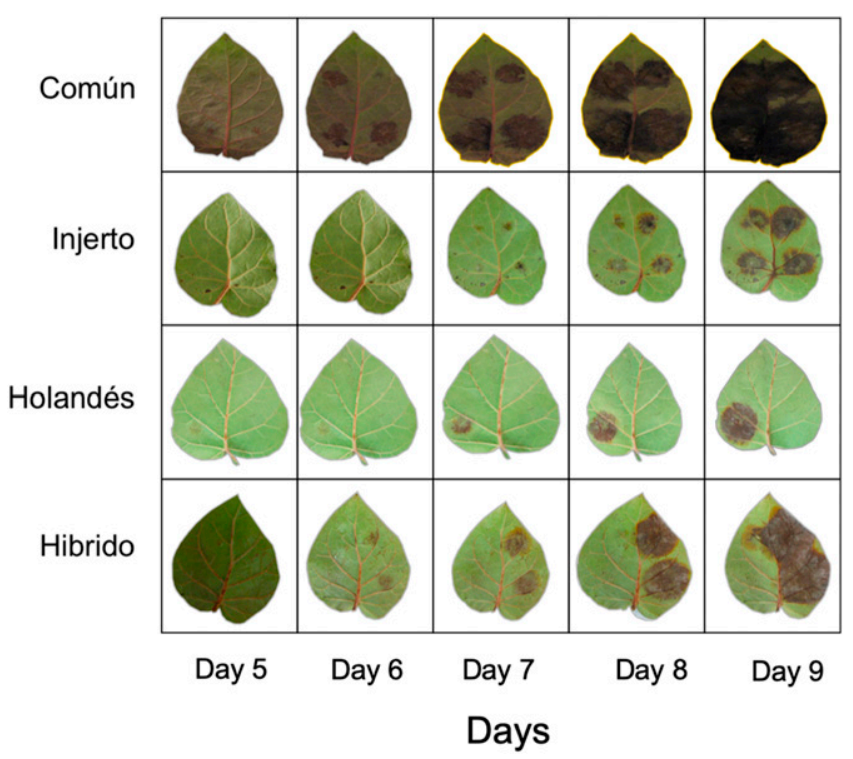

Fig. 1. Development of lesions starting at 5 days after inoculation on four tree tomato cultivars with a Phytophthora betacei isolate. successful infections, respectively. Average lesion size across isolates tested was highest for the cultivar Común at $9.7 \mathrm{~cm}^{2}$ and lowest for Holandés $7.1 \mathrm{~cm}^{2}$ (Table 3). However, Común had a negative correlation related to host colonization (lesion size) and sporangia production. For Holandés, all $P$. betacei isolates produced small lesions with low infection efficiency but had high sporangial production per lesion area.

\section{Discussion}

This is the first study on the pathogenicity of isolates of the recently described species $P$. betacei and on the susceptibility of the most commonly used tree tomato (S. betaceum) cultivars. Neither breeding strategies nor a formal breeding program on tree tomato exist in Colombia. Only some cultivars are grown in several states in the country, and therefore it is important to understand their response to the pathogen and also set the basis for a future breeding program.

The results of this study showed variation in pathogenicity of $P$. betace $i$ isolates, under controlled conditions, and differential susceptibility of four distinct $S$. betaceum cultivars. Analysis of host-pathogen interactions by original location where isolates were collected were not significant, indicating that location had no influence on pathogenicity traits evaluated in this research. Several studies have shown variation in pathogenicity of isolates of Phytophthora spp. belonging to the same clonal lineage. Delgado et al. (2013) found variation in a clonal population of $P$. infestans isolates when inoculated on a differential set of Solanum demissum clones with 11 known resistance (R) genes. These data and other research on pathogenicity of Phytophthora spp. show the importance of screening germplasm with multiple isolates when making selections for resistance in breeding programs for late blight.

From the experiments conducted in this study, it appears that at least one cultivar, Holandés, may have a major $\mathrm{R}$ gene or cluster of resistance genes, which can confer qualitative-like or partial resistance, because only 21 of 36 isolates were able to infect this cultivar (Fig. 2). Additionally, in comparison with the other cultivars evaluated, it had the longest incubation periods and the lowest infection rate (Table 3). Híbrido also showed signs of resistance, with some isolates unable to cause infection. Furthermore, it had higher incubation and latent periods than the susceptible cultivar Común (Table 3). The tree tomato cultivar Común appeared highly susceptible in that all isolates tested were able to infect it and produce large lesions. A previous study also found significant differences in susceptibility of two $S$. betaceum cultivars when inoculated with $P$. infestans sensu lato isolates in a detached leaf assay (Revelo et al. 2011).

Further studies are needed to demonstrate whether these results, obtained under controlled experiments, can be duplicated in the field. However, detached leaf assays for resistance screening have been shown to correlate well with field evaluations (Foolad et al. 2015). Inoculations of $P$. andina on tree tomato plants displayed similar lesion development under both field and laboratory conditions (Forbes et al. 2016). Additionally, investigations on aggressiveness and competitive fitness using detached leaflets of tomato and potato with

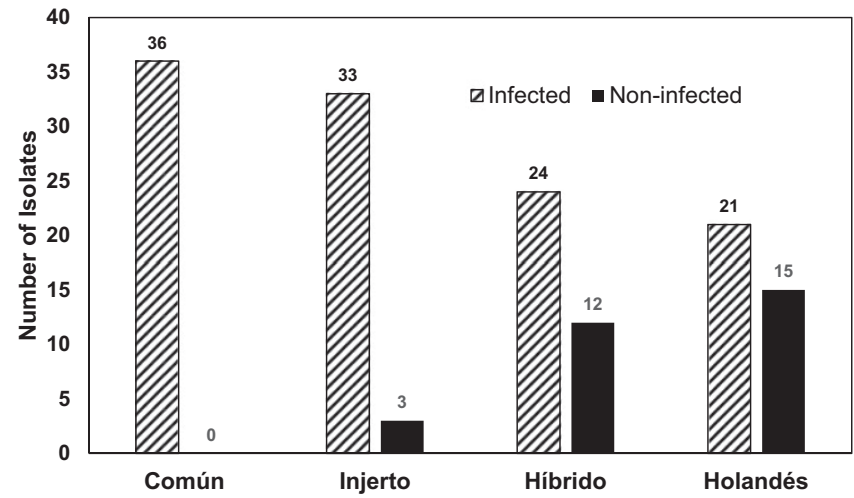

Fig. 2. Variation in the number (from 0 to 36) of Phytophthora betacei isolates able to cause infection on four different Solanum betaceum cultivars. The $y$-axis is the number of isolates able to infect, and the $x$-axis identifies the different tree tomato cultivars. 


\section{EComún DInjerto VHíbrido ĐHolandés}

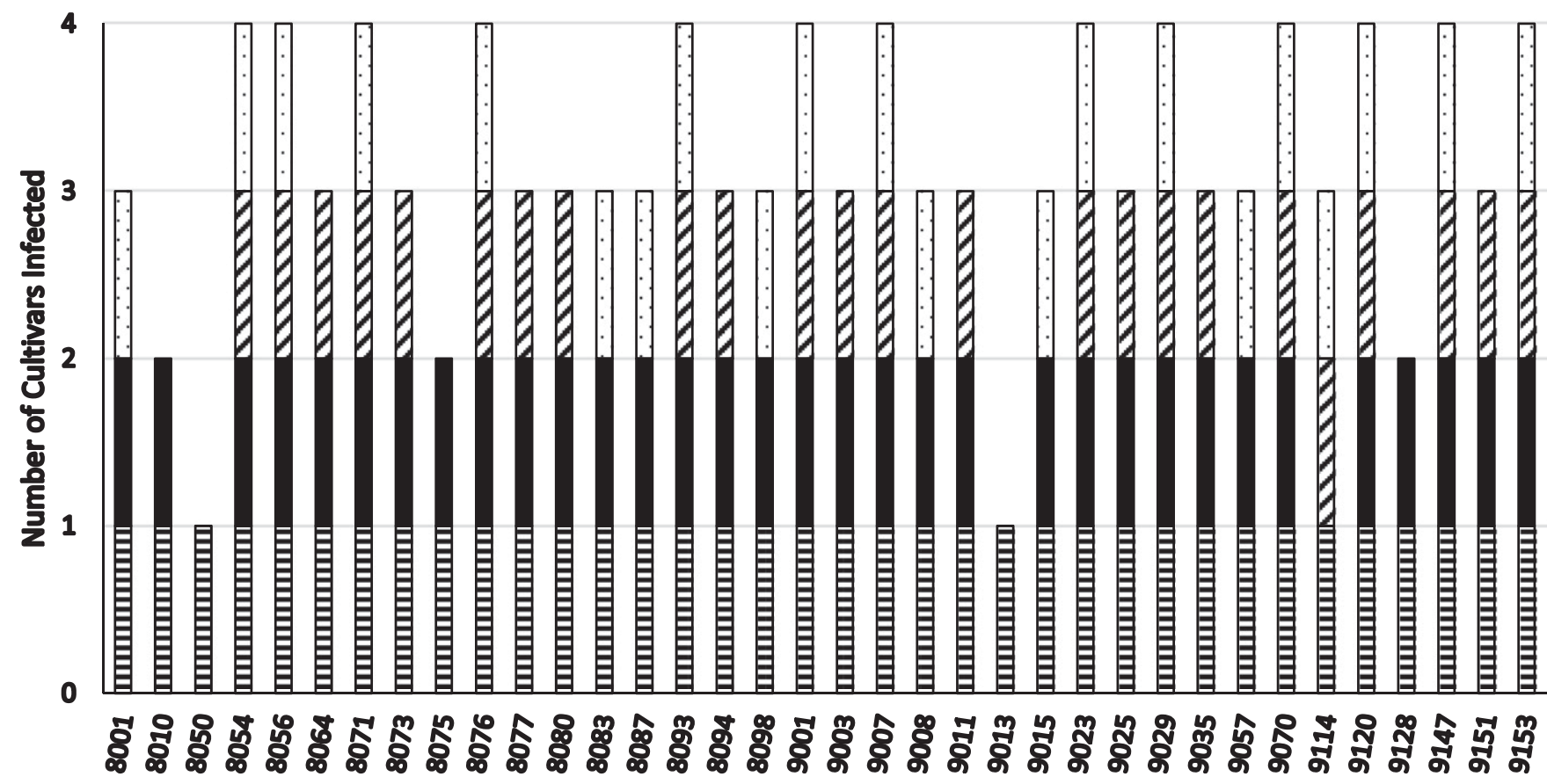

Fig. 3. Variation in pathogenicity of 36 Phytophthora betacei isolates ( $x$-axis) when inoculated on four Solanum betaceum cultivars. The $y$ axis represents the number ( 0 to 4$)$ of cultivars infected by a specific $P$. betacei isolate.

multiple isolates reported analogous results with inoculations on whole plants (Lebreton et al. 1999). Vleeshouwers et al. (1999) found a close correlation between laboratory and field assays for susceptibility and resistance but did observe some differences in detached leaf assays and field inoculations. Therefore, it is important to be aware of the limitations of inoculations conducted in the laboratory, particularly when working with new host-pathogen systems. Knowledge of the influence of pathogen populations on disease susceptibility of cultivated and wild $S$. betaceum is critical for plant breeding of resistant cultivar groups for economically viable production of this potentially promising fruit crop.

\section{Literature Cited}

Acosta-Quezada, P. G., Laborde, J. M., and Prohens, J. 2011. Variation among tree tomato (Solanum betaceum Cav.) accessions from different cultivar groups: implications for conservation of genetic resources and breeding. Gen. Res. Crop Evol. 58:943-960.

Adler, N. E., Erselius, L. J., Chacon, M. G., Flier, W. G., Ordoñez, M. E., Kroon, L. P., and Forbes, G. A. 2004. Genetic diversity of Phytophthora infestans sensu lato in Ecuador provides new insight into the origin of this important plant pathogen. Phytopathology 94:154-162.

Bonnet, J. G., and Cárdenas, J. F. 2012. Tomate de árbol (Cyphomandra betacea Sendt.). Pages 825-850 in: G. Fischer, ed. Manual Para el Cultivo de Frutales en el Trópico. Produmedos, Bogota, Colombia.

Delgado, R. A., Monteros-Altamirano, A. R., Li, Y., Visser, R. G. F., Van der Lee, T. A. J., and Vosman, B. 2013. Large subclonal variation in Phytophthora infestans populations associated with Ecuadorian potato landraces. Plant Pathol. 62:1081-1088.

Enciso-Rodríguez, F., Martínez, R., Lobo, M., and Barrero, L. S. 2010. Genetic variation in the Solanaceae fruit bearing species lulo and tree tomato revealed by Conserved Ortholog (COSII) markers. Genet. Mol. Biol. 33:271-278.

Espin, S., Gonzalez-Manzano, S., Taco, V., Poveda, C., Ayuda-Durán, B., Gonzalez-Paramas, A. M., and Santos-Buelga, C. 2016. Phenolic composition and antioxidant capacity of yellow and purple-red Ecuadorian cultivars of tree tomato (Solanum betaceum Cav.). Food Chem. 194:1073-1080.

Foolad, M. R., Sullenberger, M. T., and Ashrafi, H. 2015. Detached-leaflet evaluation of tomato germplasm for late blight resistance and its correspondence to field and greenhouse screenings. Plant Dis. 99:718-722.

Forbes, G., Gamboa, S., and Lindqvist-Kreuze, H. 2016. Identification of an A2 population of Phythophthora andina attacking tree tomato in Peru indicates a risk of sexual reproduction in this pathosystem. Plant Pathol. 65:1109-1117.
Lebreton, L., Lucas, J. M., and Andrivon, D. 1999. Aggressiveness and competitive fitness of Phytophthora infestans isolates collected from potato and tomato in France. Phytopathology 89:679-686.

Martin, F. N., Abad, Z. G., Balci, Y., and Ivors, K. 2012. Identification and detection of Phytophthora: reviewing our progress, identifying our needs. Plant Dis. 96:1080-1103.

Mideros, M. F., Turissini, D. A., Guayazán, N., Ibarra-Avila, H., Danies, G., Cárdenas, M., Myers, K., Tabima, J., Goss, E. M., Bernal, A., and Lagos, L. E. 2018. Phytophthora betacei, a new species within Phytophthora clade 1c causing late blight on Solanum betaceum in Colombia. Persoonia 41:39-55.

Murillo-Gómez, P. A., Hoyos, S., and Chavarriaga, P. 2017. Organogenesis invitro using three tissues types of tree tomato [Solanum betaceum (Cav.)]. Agron. Colomb. 35:5-11.

Nor, N. Z. N. M., Kormin, F., Fuzi, S. F. Z. M., and Bakar, M. A. L. A. 2018 Comparison of physicochemical, antioxidant properties and sensory acceptance of puree from tamarillo and tomato. J. Sci. Technol. 10:25-31.

Oliva, R. F., Kroon, L. P. N. M., Chacón, G., Flier, W. G., Ristaino, J. B., and Forbes, G. A. 2010. Phytophthora andina sp. nov., a newly identified heterothallic pathogen of solanaceous hosts in the Andean highlands. Plant Pathol. 59:613-625.

Ordoñez, M. E., Hohl, H. R., Velasco, J. A., Ramon, M. P., Oyarzun, P. J., Smart, C. D., Fry, W. E., Forbes, G. A., and Erselius, L. J. 2000. A novel population of Phytophthora, similar to P. infestans, attacks wild Solanum species in Ecuador. Phytopathology 90:197-202.

Pardo-De la Hoz, C. J., Calderón, C., Rincón, A. M., Cárdenas, M., Danies, G. López-Kleine, L., Restrepo, S., and Jiménez, P. 2016. Species from the Colletotrichum acutatum, Colletotrichum boninense and Colletotrichum gloeosporioides species complexes associated with tree tomato and mango crops in Colombia. Plant Pathol. 65:227-237.

Prohens, J., and Nuez, F. 2001. The tamarillo (Cyphomandra betacea): a review of a promising smal fruit crop. Small Fruits Rev. 1:43-68.

Revelo, E., Dorado, G., Lagos, L. E., and Burbano-Figueroa, O. 2011. Foliar virulence of isolates of Phytophthora infestans sensu lato on detached leaves of two Solanum betaceum cultivars. Trop. Plant Pathol. 36:367-373.

Rojas, P., Pardo-De la Hoz, C. J., Calderón, C., Vargas, N., Cabrera, L. A., Restrepo, S., and Jiménez, P. 2018. First report of Colletotrichum kahawae subsp. Ciggaro causing anthracnose disease on tree tomato in Cundinamarca, Colombia. Plant Dis. 102:2031.

Vleeshouwers, V. G., van Dooijeweert, W., Keizer, L. P., Sijpkes, L., Govers, F., and Colon, L. T. 1999. A laboratory assay for Phytophthora infestans resistance in various Solanum species reflects the field situation. Eur. J. Plant Pathol. 105 241-250. 